\title{
FREE $\alpha$-EXTENSIONS OF AN ARCHIMEDEAN VECTOR LATTICE AND THEIR TOPOLOGICAL DUALS
}

\author{
ANTHONY J. MACULA
}

\begin{abstract}
Arch denotes the category of Archimedean vector lattices with vector lattice homomorphisms, and $\alpha$ denotes an uncountable cardinal number or the symbol $\infty$. Arch $(\alpha)$ denotes the category of Arch objects with $\alpha$ complete Arch morphisms.
\end{abstract}

In this paper we construct, for each $L \in|\operatorname{Arch}|, \alpha$-complete extensions $L^{\prime}$ of $L$ that lift Arch morphisms from $L$ to $\alpha$-complete Arch morphisms from $L^{\prime}$. Specifically, we construct the free $\alpha$-extension and the free $\alpha$-regular extension of an Arch object $L$. By virtue of the latter, the full subcategory of $\alpha$-complete objects, in $\operatorname{Arch}(\alpha)$, is epireflective. The proofs work in Boolean algebras and recover the results obtained in $[\mathrm{K}, \mathrm{Y}$, and S]. Our proofs are different and, it can be argued, more natural.

$\mathscr{W}$ denotes the category of Arch objects with distinguished weak unit and Arch morphisms that preserve units. We exploit a certain contravariant functor $Y: \mathscr{W} \rightarrow$ Comp (the so-called Yosida functor, analogous to the Stone-space functor) from $\mathscr{W}$ to the category of compact Hausdorff spaces with continuous functions, to convert algebraic results in $\mathscr{W}$ to topological results in the topological category $\alpha-S p F i$. Specifically, we show that the Yosida "dual" of the $\mathscr{W}$-free $\alpha$-regular extension of $C(X)$ is the $\alpha$-disconnected $\alpha-S p F i$ monocoreflection of the compact space $X$, thereby showing that the full subcategory of $\alpha$-disconnected spaces, in $\alpha-S p F i$, is monocoreflective.

\section{INTRODUCTION}

$\alpha$ denotes an uncountable cardinal number or the symbol $\infty$. The meaning of $\alpha=\infty$ will be clear from the context. When we write $\alpha<\infty$ or $|A|<\infty$, where $A$ is a set, we mean that $\alpha$ or $|A|$ is an arbitrary cardinal number.

Arch denotes the category of Archimedean vector lattices with vector lattice homomorphisms. An element $u \in L \in \mid$ Arch $\mid$ is called a weak unit if the band (complete ideal) generated by $u$ is all of $L$ [LZ, dJvR]. $u$ is called a strong unit if the principal ideal generated by $u$ is all of $L$. $\mathscr{W}(\mathscr{S})$ denote the category of Arch objects with distinguished weak (strong) unit and unit preserving Arch

Received by the editors December 1, 1989 and, in revised form, May 16, 1990.

1980 Mathematics Subject Classification (1985 Revision). Primary 06A23, 06B30, 18B30, 54C10, 54G05; Secondary 06B15, 06F 15.

Key words and phrases. Free $\alpha$-regular extension, free $\alpha$-extension, $\alpha$-complete vector lattice, $\alpha$-disconnected space, Yosida space. 
morphisms. Obviously a strong unit is a weak unit, so $\mathscr{S}$ is a subcategory of $\mathscr{W}$.

An Arch morphism $\varphi: L \rightarrow M$ is called $\alpha$-complete if, for $A \subset L$ with $|A|<\alpha$, we have that $\varphi\left(\bigvee^{L} A\right)=\bigvee^{M} \varphi[A]$ whenever $\bigvee^{L} A$ exists in $L$. $\operatorname{Arch}(\alpha), \mathscr{W}(\alpha)$, and $\mathscr{S}(\alpha)$ denote the categories of Arch, $\mathscr{W}$, and $\mathscr{S}$ objects with $\alpha$-complete morphisms respectively.

An $L \in \mid$ Arch $\mid$ is called $\alpha$-complete $\bigvee^{L} A$ exists in $L$ for all bounded above $A \subset L$ with $|A|<\alpha$.

Recall $L$ is called Dedekind complete if every subset of $L$ that is bounded above has a supremum in $L$; thus $L$ is $\infty$-complete if and only if $L$ is Dedekind complete.

$L \subseteq M$ denotes that $L$ is an Arch subspace of $M$ (i.e., $L$ is a vector lattice subspace of $M$ ), while $L \subseteq \subseteq^{\alpha} M$ denotes that, in addition to $L \subseteq M$, the inclusion of $L$ into $M$ is an $\alpha$-complete Arch morphism. As usual, we reserve $\subset$ for ordinary set inclusion.

The next two definitions, as do the definitions of an $\alpha$-complete morphism and an $\alpha$-complete object, make sense in $\mathscr{W}, \mathscr{S}$, and Boolean algebras $(\equiv \mathscr{B} \mathscr{A})$, as well as in Arch .

For $L \subseteq M$, we say that $L$ is $\alpha$-full subspace in, or of, $M$ if $b=\bigvee^{M}\{A$ : $A \subset L,|A|<\alpha\}$ implies that $b \in L$ (i.e., $\left.b=\bigvee^{L} A\right)$. We call an Arch embedding $\varphi: L \hookrightarrow M \alpha$-full if $\varphi[L]$ is $\alpha$-full in $M$.

What we call an $\alpha$-full subspace of $M$, Sikorski (in $\S 23$ of [S] for Boolean algebras) calls an $\alpha$-subalgebra of $M$.

For $L \subseteq M$, we say that $L \alpha$-generates $M$ if $M$ is the smallest $\alpha$-full subspace of $M$ that contains $L$. That is, if $L^{\prime}$ is $\alpha$-full in $M$ and $L \subseteq L^{\prime}$, then $L^{\prime}=M$.

Let $L$ and $M$ be Arch objects and let $\varphi: L \hookrightarrow M$ be an Arch morphism with $M \alpha$-complete. We say the pair $(\varphi, M)$ is an Arch free $\alpha$-extension of $L$ if $\varphi[L] \alpha$-generates $M$, and each Arch morphism from $L$ into an $\alpha$ complete Arch object $N$ can be extended to an $\alpha$-complete Arch morphism from $M$ into $N$. We say the pair $(\varphi, M)$ is an Arch free $\alpha$-regular extension of $L$ if $\varphi$ is an Arch $\alpha$-complete embedding, $\varphi[L] \alpha$-generates $M$, and each $\alpha$-complete Arch morphism from $L$ into an $\alpha$-complete Arch object $N$ can be extended to an $\alpha$-complete Arch morphism from $M$ into $N$.

Analogously, one obtains the definitions of a $\mathscr{W}, \mathscr{S}$, or $\mathscr{B} \mathscr{A}$ free $\alpha$-regular extension and a $\mathscr{W}, \mathscr{S}$, or $\mathscr{B} \mathscr{A}$ free $\alpha$-extension by replacing the symbol Arch with $\mathscr{W}, \mathscr{S}$, or $\mathscr{B} \mathscr{A}$ in the above.

In $\S 3$, we indicate that, for each $L$, there is essentially, one free $\alpha$-regular extension, and one free $\alpha$-extension, of $L$.

Henceforth, $L, M$, and $N$ denote Arch objects, and maps between them are always considered to be Arch morphisms unless otherwise stated.

For $\alpha<\infty$, Yaqub shows in [Y] that every Boolean algebra has a free $\alpha$ extension. However, in [Ha], Hales shows that the free Boolean algebra on $\omega$ generators does not have a free $\infty$-extension.

The existence of the free $\alpha$-regular extension of a Boolean algebra (for $\alpha<$ $\infty)$ was first proved by Kerstan in $[\mathrm{K}]$, and subsequently, independently by Sikorski and Yaqub in $\left[\mathrm{S}_{2}\right.$ and $\left.\mathrm{Y}\right]$ respectively. In [S], Sikorski expands on the idea of free $\alpha$-regular extensions of Boolean algebras and discusses what 
he calls $(J, M, \mathfrak{m})$-extensions. Also, since in $\mathscr{B} \mathscr{A}$, the injective objects are exactly the $\infty$-complete Boolean algebras [S, 33.1], the free $\infty$-regular extension of a Boolean algebra $B$ is the Dedekind completion of $B$ [S]. Moreover, even though there are no injectives in Arch [Co], the $\infty$-regular extension of an $L \in \mid$ Arch $\mid$ is the Arch Dedekind completion of $L\left[\mathrm{BH}_{1}, \mathrm{M}_{1}\right]$.

What we do here in $\S 3$, for $\alpha<\infty$, is construct the Arch free $\alpha$-extension and the Arch free $\alpha$-regular extension of an Arch object $L$. The proofs work in $\mathscr{W}$ and $\mathscr{S}$, as well as in $\mathscr{B} \mathscr{A}$, and recover the results obtained in [K, Y, and S]. Our proofs are different, and, it can be argued, more natural.

In $\S 4$ here we exploit a certain contravariant functor $Y: \mathscr{W} \rightarrow$ Comp (the so-called Yosida functor, analogous to the Stone-space functor) from $\mathscr{W}$ to the category of compact Hausdorff spaces with continuous functions, to convert algebraic results in $\mathscr{W}$ to topological results in the topological category $\alpha-S p F i$. Specifically, we show that the Yosida "dual" of the $\mathscr{W}$-free $\alpha$-regular extension of $C(X)$ is the $\alpha$-disconnected $\alpha-S p F i$ monocoreflection of the compact space $X$, thereby showing that the full subcategory of $\alpha$-disconnected spaces, in $\alpha-S p F i$, is monocoreflective.

We begin a discussion about the Yosida functor and the category $\alpha-S p F i$.

\section{The Yosida Functor AND THE CATEgory $\alpha-S p F i$}

$X, Y$, and $Z$ denote compact Hausdorff spaces; $f, g$, and $h$ denote continuous functions; and Comp denotes the category of compact Hausdorff spaces with continuous functions.

We review the Yosida representation theory. For each $L \in|\mathscr{W}|$ there is an associated compact Hausdorff space, $Y(L)$, called the Yosida space of $L$. See [LZ, BKW, and HeR]. Also for each $\varphi: L \rightarrow M$, there is associated continuous function $Y(\varphi): Y(M) \rightarrow Y(L)$. See [HR $]$. It turns out that $Y$ is a faithful (contravariant) functor from $\mathscr{W}$ to Comp. The functor $Y$ works very much like the Stone functor from Boolean algebras to Boolean spaces. $Y(L)$, like the Stone space of a Boolean algebra, is a maximal ideal space. The elements of $Y(L)$ are ideals of $L$ that are maximal for the property of not containing the weak unit. If the weak unit is a strong unit then these ideals are the actual maximal ideals of $L$. The topology on this space is the hull-kernel topology. In fact, if we view a Boolean algebra, $B$, as a $\mathscr{W}$ object (i.e., if $L(S(B)$ ) is the locally constant real-valued functions on the Stone space $S(B)$, then $L\left(S\left(B_{1}\right)\right) \cong L\left(S\left(B_{2}\right)\right)$ iff $\left.B_{1} \cong B_{2}\right)$, the Yosida functor can be thought of as an extension of the Stone functor.

The following paragraph comes from $\left[\mathrm{BH}_{2}\right]$.

The archetypal $\mathscr{W}$ object is $C(X)$ (the ring of continuous real-valued functions on $X$ with the pointwise sup and inf). The weak unit of $C(X)$ will always be taken to be the constant function 1 . Note that the weak unit 1 is indeed a strong unit and $(C(X), \mathbf{1})$ is an $\mathscr{S}$ object. Let $D(X)$ be the set of extended real-valued continuous functions, $f: X \rightarrow[-\infty,+\infty]$, for which $f^{-1}(\mathbf{R})$ is dense in $X$. In the pointwise order, $D(X)$ is a lattice, but usually fails to be a vector space. For $f, g, h \in D(X)$, we say " $f+g=h$ in $D(X)$ " if $f(x)+g(x)=h(x)$ when $x \in f^{-1}(\mathbf{R}) \cap g^{-1}(\mathbf{R}) \cap h^{-1}(\mathbf{R})$ (which is a dense set in $X)$. It may well happen that, for particular $f, g \in D(X)$, there is no $h \in D(X)$ with $f+g=h$ in $D(X)$ (e.g., take $X=[-\infty,+\infty], f$ the obvious 
extension of $x+\sin x$, and $g$ the extension of $-x)$. However, it may well happen that a subset $L \subset D(X)$ has the property that for all $f, g \in L$ there is an $h \in D(X)$ with $f+g=h$ in $D(X)$; if $L$ is also a vector lattice under the pointwise operation in $D(X)$ and the constant function, 1 , is in $L$, then we say " $(L, \mathbf{1})$ (or just $L$ ) is a $\mathscr{W}$ object in $D(X)$ ", (e.g., $C(X)$ is a $\mathscr{W}$ object, in $D(X))$. If $X$ has the property that each dense cozero set is $C^{*}$-embedded [GJ], then $X$ is called $\omega_{1}$-quasi- $F$ (or just quasi- $F$ ) [DHH, $\mathrm{HVW}, \mathrm{BHN}, \mathrm{M}_{2}$ ]. If $X$ is $\omega_{1}$-quasi- $F$, then $(D(X), \mathbf{1}) \in|\mathscr{W}|$. See [HJ].

2.1 Theorem (see [BKW, HR1]). (a) There is a $\mathscr{W}$ isomorphism, ^ :L $\rightarrow \widehat{L} \subset$ $D(Y(L))$, onto a $\mathscr{W}$ object, $\widehat{L}$ in $D(Y(L))$, with $\hat{w}_{L}=\mathbf{1}$, and $\widehat{L}$ separates the points of $Y(L)$.

(b) If $L^{\prime}$ is a $\mathscr{W}$ object in $D(X)$ which separates the points of $X$, and for $a \in L$, if $a \mapsto a^{\prime}$ is a $\mathscr{W}$ isomorphism from $L$ to $L^{\prime}$, then there is $a$ homeomorphism $f: X \rightarrow Y(L)$ such that $a^{\prime}=\hat{a} \circ f$ for all $a \in L$.

2.1(b) is used to recognize Yosida representations.

2.2 Corollary. $Y(C(X))=X$.

Proof. In $D(X), C(X)$ satisfies $2.1(\mathrm{~b})$.

2.3 Theorem $\left[\mathrm{HR}_{1}\right]$. Let $\varphi_{i}: L \rightarrow M$ for $i=1,2$.

(a) There is a unique continuous function, $Y\left(\varphi_{1}\right): Y(M) \rightarrow Y(L)$, such that $\varphi(a)^{\wedge}=\hat{a} \circ Y\left(\varphi_{1}\right)$ for all $a \in L$.

(b) $Y$ is a faithful functor, i.e., if $\varphi_{1} \neq \varphi_{2}$, then $Y\left(\varphi_{1}\right) \neq Y\left(\varphi_{2}\right)$.

(c) $\varphi_{1}$ is one-to-one if and only if $Y\left(\varphi_{1}\right)$ is onto, and if $\varphi_{1}$ is onto, then $Y\left(\varphi_{1}\right)$ is one-to-one.

(d) Let $\gamma: C(X) \rightarrow M$ and $M \in|\mathscr{S}|$. Then $Y(\gamma): Y(M) \rightarrow X$ is one-to-one if and only if $\gamma$ is onto.

Henceforth, $L$ and $\widehat{L}$ are identified.

Thus we will consider $a \in L$ as an extended real-valued function on $Y(L)$.

The next proposition is straightforward.

2.4 Proposition. Let $f: X \rightarrow Y$. Define $f^{\prime}: C(Y) \rightarrow C(X)$ by $f^{\prime}(g)=g \circ f$ for $g \in C(Y)$ [GJ, 10.2]. Then $f^{\prime}$ is a $\mathscr{W}$ morphism and $Y\left(f^{\prime}\right)=f$.

2.5 Theorem (Banach-Stone). A function $f: X \rightarrow Y$ is a homeomorphism if and only if $f^{\prime}: C(Y) \rightarrow C(X)$ is an isomorphism in $\mathscr{W}$.

Proof. $Y\left(f^{\prime}\right)=f$. Apply $2.3(\mathrm{c})$ and $(\mathrm{d})$.

We begin a discussion of the topological category $\alpha-S p F i$. See [BHM, BHN, $\mathbf{M}_{1}, \mathbf{M}_{2}$, and $\mathrm{BH}_{3}$.

Let $\operatorname{Coz}(X)=\left\{f^{-1}(\mathbf{R} \backslash\{0\}): f \in C(X)\right\}$. A subset $V \subset X$ is said to be an $\alpha$-cozero set if

$$
V=\bigcup\left\{U_{i}: i \in I,|I|<\alpha, U_{i} \in \operatorname{Coz}(X)\right\} .
$$

Note that an $\omega_{1}$-cozero set is a cozero set. Recall that by " $|I|<\infty$ " we mean that " $|I|$ is unrestricted," so that every open set is an $\infty$-cozero set. We denote the collection of $\alpha$-cozero sets of $X$ by $\operatorname{Coz}_{\alpha}(X)$. Let $G_{\alpha} X$ denote the filter base of dense members of $\mathrm{Coz}_{\alpha}(X)$. 
A continuous function $f: X \rightarrow Y$ is called an $\alpha-S p F i$ morphism if $f^{-1}(G)$ $\in G_{\alpha} X$ whenever $G \in G_{\alpha} Y$. We thus have a topological category, denoted $\alpha-S p F i$, which consists of compact Hausdorff spaces and $\alpha-S p F i$ morphisms.

Below (2.6) is a cardinal generalization of $4.2(\mathrm{c})$ of $\left[\mathrm{BH}_{1}\right]$. See also $\left[\mathrm{M}_{2}\right]$. It tells us that the Yosida functor converts an $\alpha$-complete $\mathscr{W}$ morphism into an $\alpha-S p F i$ morphism, and, that every $\alpha-S p F i$ morphism arises from an $\alpha$ complete $\mathscr{W}$ morphism.

2.6 Lemma. $\varphi: L \rightarrow M$ is $\alpha$-complete if and only if $Y(\varphi): Y(M) \rightarrow Y(L)$ is an $\alpha$-SpFi morphism. Moreover, a function $f: X \rightarrow Y$ is an $\alpha-S p F i$ morphism if and only if $f^{\prime}(2.5)$ is $\alpha$-complete.

From this result it is routine to see that $Y$ restricted to $\mathscr{W}(\alpha)$ is a functor to $\alpha-S p F i$.

Moreover, $Y$ take $\mathscr{W}(\alpha)$ epics to $\alpha-S p F i$ monics. Recall that in a category, a morphism $e$ is called epic if $f_{1} \circ e=f_{2} \circ e$ implies that $f_{1}=f_{2}$, and a morphism $m$ is called monic if $m \circ f_{1}=m \circ f_{2}$ implies that $f_{1}=f_{2}$. We have

2.7 Lemma $\left[\mathrm{M}_{2}\right] . \varphi: L \rightarrow M$ is epic in $\mathscr{W}(\alpha)$ if and only if $Y(\varphi): Y(M) \rightarrow$ $Y(L)$ is monic in $\alpha-S p F i$.

Note that epics in $\mathscr{W}(\alpha)$ are not always surjective, nor are the monics in $\alpha-S p F i$ always injective [BHM].

A space $X$ is said to be $\alpha$-disconnected if the closure of every $\alpha$-cozero is open. It is obvious that the notions of $\infty$-disconnected and extremally disconnected (and $\omega_{1}$-disconnected and basically disconnected) are equivalent.

As the next two statements indicate, the topological concept of $\alpha$-disconnected has an algebraic counterpart. The first is a cardinal generalization of (3.3) $((\mathrm{a}) \Rightarrow(\mathrm{d}))$ of $\left[\mathrm{BH}_{1}\right]$. See also $\left[\mathrm{M}_{1}\right]$.

2.8 Lemma. Let $L \in|\mathscr{W}|$. If $L$ is $\alpha$-complete, then $Y(L)$ is $\alpha$-disconnected.

The converse of 2.8 is not true $\left[\mathrm{BH}_{1}, \mathrm{M}_{1}, \mathrm{M}_{2}\right]$. However, if we only consider $\mathscr{W}$ objects of the form $C(X)$, for compact $X$, we get the following well-known result:

2.9 Theorem (Stone-Nakano). $C(X)$ is $\alpha$-complete if and only if $X$ is $\alpha$ disconnected.

Note that one direction of 2.9 follows from 2.8 and the fact that $Y(C(X))=$ $X(2.2)$.

\section{FREE $\alpha$-EXTENSIONS}

3.1 Proposition (see $\left.\left[\mathrm{M}_{2}\right]\right)$. Let $\varphi: L \rightarrow M$. The following are equivalent.

(a) $\varphi$ is $\alpha$-complete.

(b) There is a $c \in L$ such that whenever $B \subset L,|B|<\alpha$, and $c=\bigvee^{L} B$, then $\varphi(c)=\bigvee^{M} \varphi[B]$.

(c) For $A \subset L$ with $|A|<\alpha$, we have that $\varphi\left(\bigwedge^{L} A\right)=\bigwedge^{M} \varphi[A]$ whenever $\wedge^{L} A$ exists in $L$.

Sometimes, as the next proposition indicates, the first factor of an $\alpha$-complete map is also $\alpha$-complete. As usual, $\gamma: M \hookrightarrow N$ denotes that $\gamma$ is injective. 
3.2 Proposition. Let $\varphi: L \rightarrow M$ and $\gamma: M \hookrightarrow N$. If $\gamma \circ \varphi$ is $\alpha$-complete, then $\varphi$ is $\alpha$-complete.

Proof. Let $c \in L$ and suppose $c=\bigvee^{L} A$ where $A \subset L$ and $|A|<\alpha$. We claim that $\varphi(c)=\bigvee^{M} \varphi[A]$. Suppose not. Then there is a $b \in M$ such that $\varphi(c)>b>\varphi(a)$ for all $a \in A$ (> means strictly greater than). Because $\gamma$ is injective we have $\gamma \circ \varphi(c)>\gamma(b)>\gamma \circ \varphi(a)$ for all $a \in A$. But this contradicts the assumption that $\gamma \circ \varphi$ is $\alpha$-complete. For then $\gamma \circ \varphi(c)=\bigvee^{N} \gamma \circ \varphi[A]$.

Recall from the introduction the definition of an $\alpha$-full subspace.

Note, for $L \subseteq M$, it is possible for $L$ to be $\alpha$-full, but not $\alpha$-completely embedded, in $M$.

3.3 Example. Let $X$ be a non- $\omega_{1}$-disconnected Boolean space. Then there is a cozero set $U$ such that $\bar{U}$ is not clopen. Let $L=\{f \in C(X): f[X \backslash U]=r$ for some $r \in \mathbf{R}\}$. Clearly, $L$ is $\infty$-full in $C(X)$, but $L$ is not even $\omega_{1}$-completely embedded in $C(X)$ : Since there are clopen sets $\left\{V_{n}: n \in \mathbf{N}\right\}$ and $C$ such that $U=\bigcup_{n} V_{n}$ and $U \subseteq C$, it is obvious that the sup of $\left\{\chi_{V_{n}}\right\}$ in $C(X)$ is not $1\left(\chi_{c} \geq \chi_{V_{n}}\right.$ for all $\left.n \in N\right)$. However, $\bigvee_{n}^{L} \chi_{V_{n}}=\mathbf{1}$ because, if $h \in L$ and $h \geq \chi_{V_{n}}$ for all $n \in \mathbf{N}$, then $h(x) \geq 1$ for all $x \in U$. Therefore $h(x) \geq 1$ for all $x \in \bar{U}$, but then, since $X \backslash U \cap \bar{U} \neq \varnothing$, it follows that $h \geq \mathbf{1}$.

However, if $M$ is $\alpha$-complete, then the $\alpha$-full subspaces of $M$ are easy to identify. See [S, §23]. We have

3.4 Proposition. Let $\varphi: L \hookrightarrow M$ be an embedding and suppose $M$ is $\alpha$ complete. Then $\varphi[L]$ is $\alpha$-full in $M$ if and only if both $L$ and $\varphi$ are $\alpha$ complete.

Proof. Verification is straightforward.

3.5 Proposition. Let $L \subseteq M \subseteq N$. If $L$ is $\alpha$-full in $M$ and $M$ is $\alpha$-full in $N$, then $L$ is $\alpha$-full in $N$.

Proof. Let $A \subset L,|A|<\alpha$, and $a=\bigvee^{N} A$. Since $M$ is $\alpha$-full in $N$, we have that $a \in M$ and $\bigvee^{M} A=\bigvee^{N} A$. Hence, since $L$ is $\alpha$-full in $M$, it follows that $\bigvee^{L} A=\bigvee^{M} A=\bigvee^{N} A$.

Let $S \subset L$. The smallest subspace of $L$ that contains $S$ is the intersection of all the subspaces of $L$ that contain $S$. We denote this subspace by $\langle S\rangle^{L}$, and we say that $S$ generates $\langle S\rangle^{L}$ in $L$. Moreover, $\langle S\rangle^{L}$ is the smallest subset of $L$ that contains $S$ and is closed under the finite vector lattice operations.

Now for given $\alpha$, one may ask if there is a smallest $\alpha$-complete subspace of $L$ that contains $S$ ? In general, this question does not always make sense because there may not be any $\alpha$-complete subspace of $L$ that contain $S$ (e.g., $C([0,1])$ contains no $\omega_{1}$-complete subspaces). However, there are always $\alpha$ full subspaces of $L$ that contain $S$.

Recall, for $L \subseteq M$, we say that $L \alpha$-generates $M$ if $M$ is the smallest $\alpha$ full subspace of $M$ that contains $L$. That is, if $L^{\prime}$ is $\alpha$-full in $M$ and $L \subseteq L^{\prime}$, then $L^{\prime}=M$. For $S \subseteq M$ we say $S \alpha$-generates $M$ if $\langle S\rangle \alpha$-generates $M$.

Clearly, an arbitrary intersection of $\alpha$-full subspaces of $M$ is an $\alpha$-full subspace of $M$. Therefore, for $L \subseteq M$, the subspace of $M$ which $L \alpha$-generates, denoted $\langle L\rangle_{\alpha}^{M}$, is the intersection of all the $\alpha$-full subspaces of $M$ that contain 
$L$. However, this "outside in" description does not provide much information about $\langle L\rangle_{\alpha}^{M}$. Is the inclusion of $L$ into $\langle L\rangle_{\alpha}^{M}$ epic in some sense? How big is $\langle L\rangle_{\alpha}^{M}$ ? To answer these and other questions about $\langle L\rangle_{\alpha}^{M}$ we use an "inside out" construction of $\langle L\rangle_{\alpha}^{M}$.

In what follows below, we may assume, without loss of generality, that $\alpha$ is a regular cardinal because: $\alpha^{+}$is always a regular cardinal, and the $\alpha$ completeness properties of objects and morphisms are equivalent to their respective $\alpha^{+}$-completeness properties when $\alpha$ is a singular cardinal.

3.6 Definition. Let $L \subseteq M$. Fix an $\alpha<\infty$ and define

$$
S_{\alpha}^{M}(L)=\left\{\bigvee^{M} A: A \subset L,|A|<\alpha, \bigvee^{M} A \text { exists }\right\} \text {. }
$$

For ordinals $\xi<\alpha$ we recursively define $L(\xi)^{M}$ (omitting ${ }^{M}$ when the context is clear) by setting:

$L(0)=L$,

$L(\xi)=\left\langle S_{\alpha}(L(\xi-1))\right\rangle$ if $\xi$ is not a limit ordinal,

$L(\xi)=\bigcup_{\sigma<\xi} L(\sigma)$ if $\xi$ is a limit ordinal.

Finally, we define $\langle L\rangle_{\alpha}^{M}=\bigcup_{\xi<\alpha} L(\xi)$.

Note, we also omit the superscript ${ }^{M}$ in $\langle L\rangle_{\alpha}^{M}$ whenever the context is clear.

3.7 Lemma. Let $L \subseteq M$ and $\alpha<\infty$.

(a) $\langle L\rangle_{\alpha}$ is a subspace of $M$.

(b) If two $\alpha$-complete morphisms from $\langle L\rangle_{\alpha}$ agree on $L$, they are equal. One might say the embedding of $L$ into $\langle L\rangle_{\alpha}$ is "epic for $\alpha$-complete morphisms" [H].

(c) If $L \subseteq^{\alpha} M$, then the embedding of $L$ into $\langle L\rangle_{\alpha}$ is epic in $\operatorname{Arch}(\alpha)$.

(d) $\left|\langle L\rangle_{\alpha}\right| \leq|L|^{\alpha}$.

(e) If $L \subseteq M \subseteq N$ and $M$ is $\alpha$-full in $N$, then $\langle L\rangle_{\alpha} \subseteq M$.

(f) $\langle L\rangle_{\alpha}$ is $\alpha$-full in $M$, and $L$ a-generates $\langle L\rangle_{\alpha}$.

(g) If $M$ is $\alpha$-complete, then $\langle L\rangle_{\alpha}$ is $\alpha$-complete and $\langle L\rangle_{\alpha} \subseteq^{\alpha} M$.

Proof. (a) Clear.

(b) Let $\gamma_{i}:\langle L\rangle_{\alpha} \rightarrow N$ be $\alpha$-complete with $i=1,2$, and suppose $\gamma_{1} \mid L=$ $\gamma_{2} \mid L$. We claim $\gamma_{1}\left|L(\xi)=\gamma_{2}\right| L(\xi)$ for all $\xi<\alpha$, from whence, (b) will follow. We proceed by transfinite induction. The claim is true for $L(0)=L$. Suppose it is true for all ordinals $\sigma<\xi$. If $\xi$ is a limit ordinal, it is clear from the definition of $L(\xi)$ that $\gamma_{1}\left|L(\xi)=\gamma_{2}\right| L(\xi)$. If $\xi$ is not a limit ordinal, then $L(\xi)=\left\langle S_{\alpha}(L(\xi-1))\right\rangle$. It suffices to see that $\gamma_{1}=\gamma_{2}$ on the set $S_{\alpha}(L(\xi-1))$. Let $b \in S_{\alpha}(L(\xi-1))$. Then there is a set $A \subset L(\xi-1)$ with $|A|<\alpha$ such that $b=\bigvee^{(L)_{\alpha}} A$. So $\gamma_{1}(b)=\bigvee^{N} \gamma_{1}[A]=\bigvee^{N} \gamma_{2}[A]=\gamma_{2}(b)$.

(c) Apply 3.2 and (b) above.

(d) Clearly, $\left|S_{\alpha}(L)\right|<|L|^{\alpha}$, so it follows that $\left|\left\langle S_{\alpha}(L)\right\rangle\right|<|L|^{\alpha}$. Transfinite induction gives us that for each $\xi<\alpha$, we have $|L(\xi)|<|L|^{\alpha}$, hence $\left|\langle L\rangle_{\alpha}\right|=$ $\left|\bigcup_{\xi<\alpha} L(\xi)\right| \leq|L|^{\alpha}$.

(e) Clearly, if $M$ is $\alpha$-full in $N$ and $L \subseteq M$, then $\left\langle S_{\alpha}^{N}(L)\right\rangle^{N} \subseteq M$. Transfinite induction implies that $L(\xi)^{N} \subseteq M$ for all $\xi<\alpha$, hence $\langle L\rangle_{\alpha}^{N} \subseteq M$.

(f) Let $A \subset\langle L\rangle_{\alpha}$ with $|A|<\alpha$. For each $a \in A, a \in L\left(\xi_{a}\right)$ for some $\xi_{a}<\alpha$. Therefore, $\sup _{A} \xi_{a}=\kappa<\alpha$. It follows that $A \subset L(\kappa)$. So if $b=\bigvee^{M} A$, then $b \in S_{\alpha}(L(\kappa+1)) \subset\langle L\rangle_{\alpha}$. 
To see that $L \quad \alpha$-generates $\langle L\rangle_{\alpha}$ let $L \subseteq L^{\prime} \subseteq\langle L\rangle_{\alpha}$ and suppose $L^{\prime}$ is $\alpha$ full in $\langle L\rangle_{\alpha}$. Then $L^{\prime}$ is $\alpha$-full in $M((\mathrm{c})$ here). Therefore it follows that $L^{\prime}=\langle L\rangle_{\alpha}((\mathrm{e})$ here $)$.

(g) Apply (f) here then 3.4.

3.8 Definition. For a given $L,(\varphi, M)$ is called a free $\alpha$-regular extension of $L$ if it satisfies the following conditions.

(i) $\varphi \quad M$ are $\alpha$-complete.

(ii) $\varphi[L] \alpha$-generates $M$.

(iii) For each $\alpha$-complete $\gamma: L \rightarrow N$ with $N \alpha$-complete, there is a unique $\alpha$-complete morphism $\tau: M \rightarrow N$ such that $\gamma=\mu \circ \varphi$.

We say that $(\varphi, M)$ is a free $\alpha$-extension of $L$ if it satisfies the following conditions.

(i') $M$ is $\alpha$-complete.

(ii') $\varphi[L] \alpha$-generates $M$.

(iii $\left.^{\prime}\right)$ For each $\gamma: L \rightarrow N$ with $N \alpha$-complete, there is a unique $\alpha$-complete morphism $\tau: M \rightarrow N$ such that $\gamma=\tau \circ \varphi$.

Note, conditions (iii) and (iii') respectively imply that free $\alpha$-regular extensions and free $\alpha$-extensions of $L$ are essentially unique.

Below we construct, for each $L$, the free $\alpha$-regular extension and the free $\alpha$-extension.

For a fixed $\alpha<\infty$ and $L$, let $T$ be a fixed set with $|T|=|L|^{\alpha}$. Let $A_{\alpha}(T)$ be the set of all $\alpha$-complete Arch objects which have $T$ as the underlying set. Let $I$ and $J$ be sets of morphisms defined as follows:

$$
\begin{aligned}
& I=\left\{\varphi: L \rightarrow M: M \in A_{\alpha}(T), \varphi \text { is } \alpha \text {-complete }\right\}, \\
& J=\left\{\gamma: L \rightarrow M: M \in A_{\alpha}(T)\right\} .
\end{aligned}
$$

If we let $M_{\varphi}$ and $M_{\gamma}$ be the codomains of $\varphi$ and $\gamma$ respectively, we can see that $L$ is naturally embedded in each of the products $\prod_{I} M_{\varphi}$ and $\prod_{J} M_{\gamma}$ by means of the evaluation map, e.g., $e: L \hookrightarrow \prod_{I} M_{\varphi}$ defined by $e(a)_{\varphi}=\varphi(a)$ for all $a \in L$. Since the operations in Arch products are coordinatewise it is straightforward to see that $\prod_{I} M_{\varphi}$ and $\prod_{J} M_{\gamma}$ are $\alpha$-complete, $L \subseteq \complement^{\alpha} \prod_{I} M_{\varphi}$, and $L \subseteq \prod_{J} M_{\gamma}$. Define

$$
F R_{\alpha} L=\langle L\rangle_{\alpha} \subseteq^{\alpha} \prod_{I} M_{\varphi} \quad \text { and } \quad F_{\alpha} L=\langle L\rangle_{\alpha} \subseteq \prod_{J} M_{\gamma}
$$

Note that the products in Arch and $\mathscr{W}$ are the set-theoretic products with coordinatewise operations, and the product in $\mathscr{S}$, of a set of $\mathscr{S}$-objects $M_{i}$, is obtained by first forming the product in Arch, and then taking the principal ideal generated by the element $\left(u_{i}\right)$, where $u_{i}$ is the strong unit of $M_{i}$.

3.9 Theorem. Let $i$ be the inclusion of $L$ in $F R_{\alpha} L$ and $i^{\prime}$ be the inclusion of $L$ in $F_{\alpha} L$. Then $\left(i, F R_{\alpha} L\right)$ and $\left(i^{\prime}, F_{\alpha} L\right)$ are the free $\alpha$-regular extension and the free $\alpha$-extension of $L$ respectively.

Proof. We prove that $\left(i, F R_{\alpha} L\right)$ is the free $\alpha$-regular extension of $L$. The proof that $\left(i^{\prime}, F_{\alpha} L\right)$ is the free $\alpha$-extension of $L$ is similar.

Since $F R_{\alpha} L$ is $\alpha$-complete, $F_{\alpha} L \subseteq \complement^{\alpha} \prod_{I} M_{\varphi}$, and $L \alpha$-generates $F R_{\alpha} L$ $(3.7(\mathrm{~g}),(\mathrm{f}))$, it suffices to see that any $\alpha$-complete morphism $\varphi: L \rightarrow M$, with $M \quad \alpha$-complete, can be extended to an $\alpha$-complete morphism $\bar{\varphi}: \prod_{I} M_{\varphi} \rightarrow$ 
$M$. We will show that $\bar{\varphi}$ is essentially a projection out of $\prod_{I} M_{\varphi}$. (Note, projections are $\infty$-complete.)

Let $\varphi: L \rightarrow M$ be $\alpha$-complete and let $M_{\varphi^{\prime}}=\langle\varphi[L]\rangle_{\alpha}^{M}$. Since $M_{\varphi^{\prime}}$ is $\alpha$ complete and $\left|M_{\varphi^{\prime}}\right|<|L|^{\alpha}(3.7(\mathrm{~g}),(\mathrm{d})), M_{\varphi^{\prime}}$ is isomorphic to some member of $A_{\alpha}(T)$. Therefore we can consider the morphism $\varphi^{\prime}: L \rightarrow M_{\varphi^{\prime}}$, where $\varphi^{\prime}(a)=\varphi(a)$ for $a \in L$, to be a member of $I$ (3.2). Moreover, since $M_{\varphi^{\prime}} \subseteq^{\alpha} M$ $(3.7(\mathrm{~g}))$, we need only to extend $\varphi$ to an $\alpha$-complete morphism $\bar{\varphi}: \prod_{i} M_{\varphi} \rightarrow$ $M_{\varphi^{\prime}}$. This is easily done by taking $\bar{\varphi}=\pi_{\varphi^{\prime}}$, where $\pi_{\varphi^{\prime}}$ is the $\varphi^{\prime}$ projection out of the product $\prod_{I} M_{\varphi}$. See Figure 3.1.

$$
\begin{aligned}
& \prod_{I} M_{\varphi} \\
& \alpha \cup I \\
& \pi_{\varphi^{\prime}} \\
& F R_{\alpha} L \\
& \quad \uparrow \\
& \varphi: L \longrightarrow M_{\varphi^{\prime}}=\langle\varphi[L]\rangle_{\alpha}^{M} \subseteq^{\alpha} M
\end{aligned}
$$

FIGURE 3.1

Recall in an abstract category $\mathscr{B}$, a full subcategory $\mathscr{A}$ is called an epireflective subcategory of $\mathscr{B}$ if, for each $B \in|\mathscr{B}|$, there is an $A_{B} \in|\mathscr{A}|$ and an epimorphism in $\mathscr{B}, e: B \rightarrow A_{B}$, such that for each $\mathscr{B}$ morphism, $f: B \rightarrow A$, to an $A \in|\mathscr{A}|$, there exists a (necessarily) unique $\mathscr{A}$ morphism, $\bar{f}: A_{B} \rightarrow A$, satisfying $f=\bar{f} \circ e .\left(e, A_{B}\right)$ is called the $\mathscr{A}$ epireflection of $B$. (Note, epireflections are essentially unique.)

The existence of the free $\alpha$-regular extension and 3.7(c) together imply:

3.10 Theorem. In $\operatorname{Arch}(\alpha)$, full subcategory of $\alpha$-complete objects is epireflective, and, for each $L,\left(i, F R_{\alpha} L\right)$ is the $\alpha$-complete epireflection of $L$ in $\operatorname{Arch}(\alpha)$.

Note all the results (in, and about, $\operatorname{Arch}$ and $\operatorname{Arch}(\alpha)$ ) of this section have analogs in $\mathscr{W}, \mathscr{W}(\alpha), \mathscr{S}, \mathscr{S}(\alpha), \mathscr{B} \mathscr{A}$ and, $\mathscr{B} \mathscr{A}(\alpha)$. Moreover, the proofs, are essentially identical to those in $\operatorname{Arch}$ and $\operatorname{Arch}(\alpha)$. Most importantly, for each $\mathscr{W}($ and $\mathscr{S})$ object, there is, in $\mathscr{W}($ and $\mathscr{S})$, a free $\alpha$-regular extension and a free $\alpha$-extension. And, as in $\operatorname{Arch}(\alpha)$, we have the following:

3.11 Theorem. In $\mathscr{W}(\alpha)$ [and $\mathscr{S}(\alpha)$ ], the full subcategory of $\alpha$-complete objects is epireflective, and, for each $L \in|\mathscr{W}|[L \in|\mathscr{S}|]$, the $\mathscr{W}[\mathscr{S}]$ free $\alpha$-regular extension $\left(i, \mathscr{W}-F R_{\alpha} L\right)\left[\left(i, \mathscr{S}-F R_{\alpha} L\right)\right]$ is the $\alpha$-complete epireflection of $L$ in $\mathscr{W}(\alpha)[\mathscr{S}(\alpha)]$.

\section{4. $\alpha$-DISCONNECTED $\alpha$-SpFi COREFLECTIONS}

Consider the $\mathscr{W}$-free $\alpha$-regular extension $\left(i, F R_{\alpha} C(X)\right)$, and the $\mathscr{W}$-free $\alpha$-extension $\left(i^{\prime}, F_{\alpha} C(X)\right)$, of $C(X)$. Let $m_{\alpha} X=Y\left(F R_{\alpha} C(X)\right)$ and $M_{\alpha} X=$ 
$Y\left(F_{\alpha} C(X)\right) \cdot\left(m_{\alpha} X, Y(i)\right)$ and $\left(M_{\alpha} X, Y\left(i^{\prime}\right)\right)$ are preimages of $X$. See Figures 4.1 and 4.2.

$$
\begin{aligned}
& C(X) \quad \stackrel{i}{\hookrightarrow} \quad F R_{\alpha} C(X) \\
& X=Y(C(X)) \stackrel{Y(i)}{\longleftarrow} Y\left(F R_{\alpha} C(X)\right)=m_{\alpha} X \\
& \text { FIGURE } 4.1 \\
& C(X) \quad \stackrel{i^{\prime}}{\hookrightarrow} F_{\alpha} C(X) \\
& X=Y(C(X)) \stackrel{Y\left(i^{\prime}\right)}{\longleftarrow} \quad Y\left(F_{\alpha} C(X)\right)=M_{\alpha} X
\end{aligned}
$$

FIGURE 4.2

Let $m_{\alpha}=Y(i)$ and $M_{\alpha}=Y\left(i^{\prime}\right)$. We have the following:

4.1 Theorem. Let $\alpha<\infty$.

(a) $m_{\alpha} X$ and $M_{\alpha} X$ are $\alpha$-disconnected.

(b) In $\alpha-S p F i, m_{\alpha}$ is monic.

(c) If $f: Y \rightarrow X$ is an $\alpha-S p F i$ and $Y$ is $\alpha$-disconnected, then there is a unique $\alpha-S p F i$ morphism $\bar{f}: Y \rightarrow m_{\alpha} X$ such that $f=m_{\alpha} \circ \bar{f}$.

(d) If $h: Y \rightarrow X$ is continuous and $Y$ is $\alpha$-disconnected, then there is a unique $\alpha-S p F i$ morphism $\bar{h}: Y \rightarrow M_{\alpha} X$ such that $h=M_{\alpha} \circ \bar{h}$.

Proof. (a) $F R_{\alpha} C(X)$ and $F_{\alpha} C(X)$ are $\alpha$-complete so $m_{\alpha} X$ and $M_{\alpha} X$ are $\alpha$-disconnected (2.8).

(b) $i$ is epic in $\mathscr{W}(\alpha)$; thus $m_{\alpha}$ is monic in $\alpha-S p F i$ (2.7).

(c) $f^{\prime}: C(X) \rightarrow C(Y)$ is an $\alpha-S p F i$ morphism (2.4 and 2.6). And since $C(Y)$ is $\alpha$-complete (2.9), there is a unique $\alpha$-complete $\mathscr{W}$ morphism $\bar{f}^{\prime}$ : $F R_{\alpha} C(X) \rightarrow C(Y)$ such that $f^{\prime}=\bar{f}^{\prime} \circ i$. Hence $f=Y\left(f^{\prime}\right)=Y\left(\bar{f}^{\prime} \circ i\right)=$ $Y(i) \circ Y\left(\bar{f}^{\prime}\right)=m_{\alpha} \circ Y\left(\bar{f}^{\prime}\right)$. Take $\bar{f}=Y\left(\bar{f}^{\prime}\right)$. $\bar{f}$ is an $\alpha-S p F i$ morphism (2.6).

(d) The proof of (d) is similar to that of (c).

Let $\mathscr{B}$ be a category and $\mathscr{A}$ a subcategory of $\mathscr{B}$. We call $\mathscr{A}$ a monocoreflective subcategory of $\mathscr{B}$ if, for each $B \in|\mathscr{B}|$, there is a $A_{B} \in|\mathscr{A}|$ and a monic in $\mathscr{B}, m_{B}: A_{B} \rightarrow B$, such that for each $\mathscr{B}$ morphism, $f: A \rightarrow B$, from an $A \in|\mathscr{A}|$, there is a (necessarily) unique $\mathscr{A}$ morphism, $\bar{f}: A \rightarrow A_{B}$, satisfying $f=m_{B} \circ \bar{f} .\left(A_{B}, m_{B}\right)$ is called an $\mathscr{A}$ monocoreflection of $B$. (Note, monocoreflections are essentially unique.)

4.2 Theorem. In $\alpha$-SpFi, the full subcategory of $\alpha$-disconnected spaces is monocoreflective, and for each $X,\left(m_{\alpha} X, m_{\alpha}\right)$ is the $\alpha$-disconnected monocoreflection of $X$ in $\alpha-S p F i$.

Proof. Apply 4.1(b) and (c).

4.3 Remark. Let $\mathrm{Ba}(X)$ be the $\sigma$-algebra of Baire sets of $X$ and let $\mathscr{Z}(X)$ be the $\sigma$-ideal generated by the nowhere dense zero-sets of $X$. In $\left[\mathrm{BH}_{3}\right]$ it is shown that $M_{\omega_{1}} X$ is the Stone space of $\mathrm{Ba}(X)$, and $m_{\omega_{1}} X$ is the Stone space of the quotient $\mathrm{Ba}(X) / \mathscr{Z}(X)$. Also, for $X$, the Stone space of the Borel sets modulo the meager Borel sets is called the absolute or Gleason cover, $E X$, of $X$ 
[PW], and $m_{\infty} X \cong E X$ [BHM, W]. See also [M $\left.\mathrm{M}_{1}, \mathrm{M}_{2}\right]$. Can $M_{\alpha} X$ and $m_{\alpha} X$, for general $\alpha$, be represented in a similar fashion? I do not know the answer for arbitrary $X$, however, if $X$ is $\alpha$-cozero complemented, the answer is "yes" for $m_{\alpha} X\left[\mathrm{M}_{3}\right] . X$ is $\alpha$-cozero complemented if, for each $U \in \mathrm{Coz}_{\alpha}(X)$, there is a $V \in \mathrm{Coz}_{\alpha} X$ such that $U \cup V$ is dense in $X$, and $U \cap V=\varnothing$.

\section{REFERENCES}

[B] G. Birkhoff, Lattice theory, Amer. Math. Soc. Colloq. Publ. Amer. Math. Soc., Providence, R. I., 3rd ed., 2nd printing, 1973.

$\left[\mathrm{BH}_{1}\right]$ R. N. Ball and A. W. Hager, Epicompletion of Archimedean l-groups and vector lattices with weak unit, J. Austral. Math. Soc. Ser. A 48 (1990), 25-56.

$\left[\mathrm{BH}_{2}\right] \_$, Epicomplete archimedean l-groups and vector lattices, Trans. Amer. Math. Soc. 322 (1990), 459-478.

[BH] - Characterization of epimorphisms in Archimedean l-groups and vector lattices, Chapter 8 Lattice-Ordered Groups, Advances and Techniques, (A. Glass and W. C. Holland, eds.), Kluwer, Dordrecht, 1989.

$\left[\mathrm{BH}_{4}\right] \ldots$, Application of spaces with filters to Archimedean l-groups, Proc. Conf. on Ordered Algebraic Structures, Curaçao, 1988 (J. Martinez, ed.), Kluwer, Dordrecht, 1989.

[BHM] R. N. Ball, A. W. Hager, and A. J. Macula, An $\alpha$-disconnected space has no proper monic preimage, Topology Appl. (to appear).

[BHN] R. N. Ball, A. W. Hager, and C. Neville, The $\kappa$-ideal completion of an Archimedean 1 group and the $\kappa$-quasi- $F$ cover of a compact space, General Topology Appl., Proc. Northeast Topology Conf., Wesleyan Univ., 1988 (R.M. Shortt, ed.), Marcel Dekker, New York, 1990, pp. 7-50.

[BKW] A. Bigard, K. Keimel and S. Wolfenstein, Groupes et anneaux réticules, Lecture Notes in Math., vol. 608, Springer-Verlag, Berlin, Heidelberg, and New York, 1977.

[C] H. B. Cohen, The $\kappa$-extremally disconnected spaces as projectives, Canad. J. Math. 16 (1964), 253-260.

[Co] P. Conrad, Minimal vector lattice covers, Bull. Austral. Math. Soc. 4 (1971), 35-39.

[DHH] F. Dashiell, A. W. Hager and M. Henriksen, Order-Cauchy completions of rings and vector lattices of continuous functions, Canad. J. Math. 32 (1980), 657-685.

[dJvR] E. DeJonge and A. C. M. Van Rooij, Introduction to Riesz spaces, Math. Centre Tracts, no. 78, Amsterdam, 1977.

[G] A. M. Gleason, Projective topological spaces, Illinois J. Math. 2 (1958), 482-489.

[GJ] L. Gillman and M. Jerison, Rings of continuous functions, Van Nostrand, Princeton, N. J., 1960.

[H] A. W. Hager, Boolean algebras with $\alpha$-complete morphisms, unpublished manuscript.

$\left[\mathrm{HR}_{1}\right]$ A. W. Hager and L. C. Robertson, Representing and ringifying a Riesz space, Symposia Math. 21 (1977), 411-431.

$\left[\mathrm{HR}_{2}\right]+$, On imbedding into a ring of an Archimedean lattice-ordered group, Canad. J. Math. 31 (1979), 1-8.

[Ha] A. W. Hales, On the non-existence of free complete boolean algebras, Fund. Math. 54 (1964), 45-66.

[HJ] M. Henriksen and D. G. Johnson, On the structure of a class of archimedean lattice-ordered algebras, Fund. Math. 50 (1961), 73-94.

[HVW] M. Henriksen, J. Vermeer, and R. G. Woods, Quasi-F covers of Tychonoff spaces, Trans. Amer. Math. Soc. 303 (1987), 779-803.

[HS] H. Herrlich and G. Strecker, Category theory, Allyn and Bacon, Boston, Mass., 1973.

[HeR] E. Hewitt and K. A. Ross, Abstract harmonic analysis. I, Springer-Verlag, Berlin, 1963.

[K] J. Kerstan, Tensorielle Erweiterungen distributiver Verbände, Math. Nachr. 22 (1960), 1-20.

[LZ] W. Luxemburg and A. Zaanen, Riesz spaces, Vol. I, North-Holland, Amsterdam, 1971. 
[M $M_{1}$ A. J. Macula, Thesis, Wesleyan Univ., 1989.

$\left[\mathrm{M}_{2}\right] \quad-, \alpha$-Dedekind complete Archimedean vector lattices $v s, \alpha$-quasi-F spaces, Topology Appl. (to appear).

[M $\left.\mathrm{M}_{3}\right]-$ Monic sometimes means $\alpha$-irreducible, General Topology and its Appl., Vol. 134, (S. J. Andima et al., ed.), Marcel Dekker, New York, 1991, pp. 239-260.

[PW] J. R. Porter and R. G. Woods, Extensions and absolutes of Hausdorff spaces, Springer-Verlag, New York, 1988.

[S] R. Sikorski, Boolean algebra, 3rd ed., Springer-Verlag, Berlin, 1969.

[ $\left.\mathrm{S}_{2}\right] \quad \ldots$, On extensions and products of boolean algebras, Fund. Math. 53 (1963), 99-116.

[W] R. G. Woods, Covering properties and coreflective subcategories, Proc. CCNY Conf. on Limits 1987, Ann. N. Y. Acad. Sci. (to appear).

[Y] K. Yosida, On the representation of a vector lattice, Proc. Imp. Acad. Tokyo 18 (1942), 339-342.

Department of Mathematics, Wesleyan University, Middletown, Connecticut 06457 